\title{
La espiral del conocimiento en la redacción de artículos científicos
}

\author{
María del Rosario Demuner-Flores, Rosa María Nava Rogel \& \\ Patricia Mercado-Salgado \\ Universidad Autónoma del Estado de México \\ E-mail: demuner7@yahoo.com/pat_mersal@yahoo.com
}

\begin{abstract}
Resumen
El último reto de toda investigación es comunicar los hallazgos encontrados de manera efectiva. Sin embargo, en las áreas económico administrativas, muchas veces no se comunica lo encontrado, ya sea porque lo encontrado no tiene una base teórica sustentable, porque no hay congruencia entre el título, el objetivo del trabajo y las conclusiones, porque los resultados encontrados no respaldan las conclusiones, porque no se explica

conocimiento o porque no se comunica una sola idea de manera sencilla e impactante. Este trabajo propone un método de gestión del conocimiento basados en la espiral del conocimiento (Nonaka y Takeuchi, 1995) que ayude a la elaboración de artículos científicos, a partir del análisis de las deficiencias encontradas en algunas investigaciones enviadas a una Revista de Administración de una universidad mexicana de alcance internacional.
\end{abstract} la aportación que hace el documento al

Palavras-clave: Conocimiento; artículos científicos; revistas científicas; divulgación.

\begin{abstract}
The last challenge of any investigation is to communicate the findings effectively. However, the administrative economic areas, often does not communicate the findings, either because it found no sustainable theoretical basis, no congruence between the title, the aim of the work and findings, the results do not support the conclusions, the contribution to

one idea so simple and powerful does not communicate. This paper proposes a method of knowledge management based on knowledge spiral (Nonaka and Takeuchi, 1995) to assist in the preparation of scientific papers, from the analysis of the deficiencies found in some research sent to Mexican Journal of Management of international scope.
\end{abstract} knowledge is not explained or because 
Keywords: Knowledge; scientific articles; scientific journals; disclosure.

\section{Introducción}

T A divulgación de la ciencia mediante la publicación de artículos científiL cos en revistas indexadas es una necesidad de cada investigador. Aunque existe una gran aportación tendiente a enseñar a escribir este tipo de documentos, se advierten rechazos de los mismos cuando éstos participan en un proceso de revisión por pares académicos, como el comúnmente usado en las revistas especializadas e indexadas. El peer review, es una actividad que toda revista científica emplea para su proceso editorial, su objetivo es precisamente, avalar la calidad de la investigación presentada para su posible publicación.

Los autores esperan un veredicto de aceptación, sin embargo, si éste implica un rechazo en una revista con una alta tasa de aceptación, donde existe mucha competencia y donde los árbitros argumentan su decisión exponiendo ideas de mejora, estas causas de rechazo se convierten en oportunidades que el autor considera para fortalecer su artículo. Cuando el árbitro condiciona el artículo es porque el autor tiene la oportunidad de considerar las observaciones que se le hicieron, y si contesta adecuadamente, quiere decir que su artículo está entre aquellos no aceptados inicialmente y su probabilidad de aceptación es del 50\%, la decisión final dependerá enteramente de la calidad de los cambios realizados y las respuestas ofrecidas a las cuestiones planteadas (Bermejo, 2002)

La calidad en los artículos, muchas veces es cuestionada porque lo que se comunica no tiene una base teórica sustentable, o se advierten incongruencias entre el título, el objetivo, las preguntas de investigación y las hipótesis; por otra parte, los resultados encontrados no respaldan las conclusiones. Adicionalmente la calidad se demerita debido al mal uso de la ortografía y la gramática.

Muchos de los estudios empíricos que se han centrado en los contextos de investigación y desarrollo similares, han tendido a utilizar diferentes medidas, con poca preocupación para la triangulación con estudios previos (Lane, Koka y Pathak, 2006). Esto ha dado lugar a un patrón disperso de la acumulación del conocimiento en el que los estudios son estadísticamente significativos, 
pero teóricamente fragmentados (Tsang y Kwan, 1999), un problema común en la mayoría de las áreas de investigación de la organización (Lane, Koka y Pathak, 2006).

Entre las principales causas de rechazo de un artículo se encuentran: falta de una hipótesis clara, ambición excesiva y falta de foco, exposición de un problema nuevo de poca importancia, falta de preparación o investigación antes de someter el manuscrito a su revisión para publicación, mal diseño del caso de aplicación, aplicabilidad sobre generalizada de los hallazgos y mala escritura (Fisher y Powers 2004).

Por su parte Lane, Koka, Pathak (2006:859) mencionan algunos aspectos que hacen que se demerite la calidad de las publicaciones:

- Las presiones para publicar han creado un desincentivo para que los investigadores puedan invertir el tiempo necesario para desarrollar una comprensión mas profunda de cualquier constructo.

- La reticencia de las revistas científicas en ciencias sociales a publicar estudios de réplica han logrado desincentivar aún mas.

- Las presiones de saltar de investigaciones emergentes para publicar rápidamente y evitar réplicas.

Aunque existe abundante literatura sobre cómo hacer un artículo científico, metodologías para realizar la investigación y normas editoriales que cada revista establece para aceptar artículos, esto no basta, el investigador debe encontrar su propio estilo para organizar y plasmar sus ideas. Es decir, debe adoptar un método adecuado a sus propias habilidades. Grawitz (1974) plantea que un método puede entenderse en cuatro formas: 1) sentido filosófico, siendo éste el nivel más alto de abstracción donde el método denota los procedimientos lógicos que debe seguir el investigador para acercarse a la verdad y verificarla; 2) el método también se refiere a la actitud que adopta el investigador respecto al objeto de estudio, por lo que en este nivel el método orienta la forma en que se organiza y lleva a cabo la investigación; 3) el sentido que adquiere el método es por su vinculación con un intento de explicación, por lo que la posición filosófica seleccionada por el investigador influye en el método a seguir en la investigación; y 4) un método propio acorde con el campo de conocimiento del investigador.

En este sentido, el presente trabajo tiene como objetivo aportar un método aplicado a trabajos de las disciplinas económico administrativas con el fin de socializar, externalizar, combinar e internalizar el conocimiento. Para ello, el 
trabajo presenta en primer lugar, un marco teórico que describe el modelo de la espiral del conocimiento; en segundo lugar se muestra cómo se desarrolló el estudio descriptivo, para finalmente presentar la propuesta seguida de algunas reflexiones finales.

\section{Fundamento Teórico}

Nonaka y Takeuchi (1995) explican el papel protagónico de la empresa para generar las condiciones que permiten la creación, difusión y aplicación del conocimiento a partir de la intención, autonomía, fluctuación y caos creativo, redundancia y variedad de requisitos. A través del modelo que proponen: "la espiral del conocimiento", se enfatiza y ubica el desarrollo del conocimiento en cuatro etapas.

El modelo inicia en la socialización del conocimiento con la creación de un campo de interacción que permite que los investigadores compartan sus experiencias y modelos mentales. En segundo lugar, la externalización se da a partir de un diálogo o reflexiones colectivas significativas, en los que el uso de una metáfora o una analogía apropiadas ayudan a los investigadores a enunciar el conocimiento tácito oculto, que de otra manera resulta difícil de comunicar. En tercer lugar, la combinación da comienzo con la distribución por redes del conocimiento recién creado y el conocimiento existente de otras fuentes, cristalizándolos así en un nuevo producto. Y en cuarto lugar, la internalización se origina en aprender haciendo. Pero el conocimiento no termina en la cuarta etapa, en ella surgen nuevas inquietudes, perspectivas, avances, dominios que hacen que el proceso se re inicie con la socialización del conocimiento.

Para que los investigadores capten, compartan, organicen y utilicen los conocimientos para lograr un trabajo de investigación que cuente con los requisitos que exigen las revistas científicas, deben implementar un procedimiento propio, que incorpore el desarrollo de una aministración activa y consciente de crear, diseminar, evolucionar y aplicar el conocimiento a fines estratégicos (Bedrow y Lane, 2003).

Si bien existe vasta literatura que aporta modelos para gestionar el conocimiento, para este estudio usaremos el modelo "La espiral de conocimiento" aportado por Nonaka y Takeuchi (1995) que identifica cuatro tipos de conocimiento: 
1. El conocimiento tácito es un conocimiento personal que está enraizado en la acción individual y en la experiencia, así como en los ideales, valores o emociones que el individuo adopta (Nonaka y Takeuchi, 1995).

2. El conocimiento explícito es el fruto de un proceso de aprendizaje; su manifestación se da a través de un lenguaje formal y sistemático que puede ser escrito, auditivo o visual, y se recopila y comparte mediante datos, fórmulas, especificaciones y manuales (Byosiere, 1999). Este tipo de conocimiento es apropiable y transmisible, a su vez está abierto a la participación y colaboración de los individuos ya que se encuentra materializado en soportes de fácil acceso, salvo que esté protegido mediante patentes (Osterloh y Frey, 2000).

3. El conocimiento individual se genera en la persona misma y puede ser el sustento del conocimiento colectivo.

4. El conocimiento colectivo es compartido por los miembros de la comunidad, y por tanto no depende de ningún individuo en concreto.

\section{Metodología}

Toda investigación tiene la responsabilidad y el compromiso de ser difundida, de comunicar los hallazgos encontrados de manera efectiva de tal forma que ayude a la generación de conocimiento y/o a la solución de problemas de la sociedad. Con el fin de aportar ideas de mejora en la elaboración de artículos científicos y disminuir el índice de rechazo, se desarrolla un estudio descriptivo que explica la experiencia que ha tenido una revista científica con los artículos que ha recibido para su publicación, desde su creación hasta la última publicación.

La unidad de análisis está representada por los artículos que ha recibido la revista desde su reciente creación (2012) a la última publicación de 2014. Se trata de una revista electrónica cuyo objetivo es crear un espacio que impulse, genere y promueva actividades de investigación y reflexión en el área académico administrativa. Cuenta con ISSN y es reconocida por cuatro índices: Dialnet, Latindex, CLASE y BASE Biblioteca de la Universidad de Bielefeld. En su página Web presenta las normas editoriales, así como los números publicados. 


\section{Resultados}

La revista publicó 8 números hasta 2014, recibió en total 80 artículos de los cuales el $26 \%$ fueron rechazados desde la revisión de escritorio, ya sea porque el contenido no correspondía a la temática que maneja la revista, por la poca relevancia del tema, por el incumplimiento en la forma, aunado a alguna otra debilidad como el tratamiento metodológico, la revisión teórica, antigüedad en las fuentes consultadas, presentación y discusión desordenada de los resultados, entre otras.

Respecto a los artículos rechazados por los árbitros, la tasa es de $18 \%$ que al sumarse con los rechazados por el editor, en su revisión de escritorio, la tasa de rechazo de la revista alcanza el $44 \%$.

En total fueron enviados a revisión peer view 59 artículos, de los cuáles 49 fueron aceptados, siendo la tasa de aceptación el 83\%, cabe hacer mención que algunos de estos artículos fueron condicionados a algunas pequeñas modificaciones, pero que al re enviarlos en la segunda ronda a los árbitros, ellos dieron el voto de aceptación (Ver tabla 1).

Como puede observarse el índice de rechazo es del 17\%. Las causas de rechazo de parte de los pares académicos que se identificaron, versan en los siguientes términos:

1. Falta de congruencia entre el título, el objetivo del trabajo y las conclusiones.

2. Escaza revisión teórica

3. Los resultados encontrados no respaldan las conclusiones

4. Metodología débil

5. Falta de argumentación

6. Poca o nula originalidad

7. Inadecuado tratamiento de los datos 
Tabla 1. Artículos recibidos, aceptados y rechazados

\begin{tabular}{lccccc} 
& 2014 & 2013 & 2012 & Total & \\
\hline Números publicados & 3 & 3 & 2 & 8 & 15 \\
Envíos totales & 37 & 25 & 18 & 80 & $100 \%$ \\
Rechazados por incumplir normas editoriales & 13 & 5 & 3 & 21 & $26 \%$ \\
Revisión por pares & 24 & 20 & 15 & 59 & $100 \%$ \\
Aceptados & 20 & 16 & 13 & 49 & $83 \%$ \\
Rechazados por pares & 4 & 4 & 2 & 10 & $17 \%$ \\
\hline
\end{tabular}

Fuente: elaboración propia.

\section{Método propuesto para gestionar el conocimiento en investigación}

Con el fin de mostrar un método que ayude al investigador a organizar y estructurar su conocimiento, se propone adoptar el aquí sugerido, mismo que se retoma de Demuner, Nava y Sandoval (2015) y cuyo sustento teórico es el modelo de la Espiral del conocimiento de Nonaka y Takeuchi (1995).

El conocimiento se crea cuando se produce una transformación del saber tácito del investigador en explícito a nivel grupal y organizativo; cada uno de los miembros de tales colectivos lo interiorizan, convirtiéndolo de nuevo en tácito (Nonaka y Takeuchi, 1995).

La espiral del conocimiento enfatiza y ubica el desarrollo del conocimiento en cuatro etapas. Inicia en la socialización del conocimiento con la creación de un campo de interacción que permite que los miembros del equipo compartan sus experiencias y modelos mentales. En segundo lugar, la externalización se da a partir de un diálogo o reflexiones colectivas significativas, en los que el uso de una metáfora o una analogía apropiadas ayudan a los miembros a enunciar el conocimiento tácito oculto, que de otra manera resulta difícil de comunicar. En tercer lugar, la combinación da comienzo con la distribución por redes del conocimiento recién creado y el conocimiento existente de otras secciones de la organización, cristalizándolos así en un nuevo producto, servicio o sistema administrativo. Y en cuarto lugar, la internalización se origina en aprender haciendo. Pero el conocimiento no termina en la cuarta etapa, en ella surgen nuevas inquietudes, perspectivas, avances, dominios que hacen que el proceso se re inicie con la socialización del conocimiento. 


\section{Socialización}

Consiste en pasar de un conocimiento tácito a otro conocimiento tácito. Se da cuando un investigador adquiere conocimiento ya sea por algún tipo de comunicación, por simple observación, imitación o práctica. Es un proceso en el que el investigador adquiere conocimiento tácito al leer, estudiar, compartir experiencias y pensamientos con otros investigadores o con algún grupo de interés, de manera que incrementa su saber y llega a conseguir niveles cercanos a los del emisor.

Para conseguir tales resultados se realizan dos actividades clave claramente diferenciadas (Nonaka y Konno, 1998), la primera, captar conocimiento a través de la interrelación con investigadores externos, grupos o centros de investigación, e internos que son colegas de la misma organización, línea de investigación o disciplina, procedente de la proximidad física o de la interacción virtual; y la segunda, diseminar el conocimiento, es decir, transferirlo a otro individuo como una exposición del tema en un salón de clases, un congreso o alguna reunión formal. En este proceso el investigador puede adquirir conocimiento tácito directamente de otros sin necesariamente usar el lenguaje (Ver tabla 2).

Medios que se pueden emplear para socializar el conocimiento:

a) Desarrollo de espacios para compartir experiencias y habilidades

b) La observación, la imitación y la práctica.

c) Exposiciones orales, discusiones detalladas, como un mecanismo con el cual los individuos buscan la armonía al involucrarse tanto en experiencias corporales como mentales.

d) Aprendizaje en equipo. La interacción con colegas antes del desarrollo del producto de investigación y después de haberlo publicado, es un proceso inter $\neg$ minable de intercambio de experiencias, habilidades y de creación de ideas para mejorar. 
Tabla 2.Proceso de socialización

\begin{tabular}{ll} 
Actividad & Descripción \\
\hline $\begin{array}{l}\text { Reconocer al otro como per- } \\
\text { sona }\end{array}$ & $\begin{array}{l}\text { Se utiliza para construir un campo de interacción, reco- } \\
\text { nociendo las distintas características de los individuos } \\
\text { como antecedentes, experiencia profesional dentro y fu- } \\
\text { era de la organización, metas individuales, perspectivas } \\
\text { y motivaciones. }\end{array}$ \\
$\begin{array}{l}\text { Conocimiento del otro para } \\
\text { generar confianza mutua }\end{array}$ & $\begin{array}{l}\text { Se trata de construir un entorno empático y de mutua } \\
\text { confianza, por lo que las emociones, sentimientos y cre- } \\
\text { encias de cada persona tendrían que ser compartidos. }\end{array}$ \\
Interactuación cara a cara & $\begin{array}{l}\text { Los investigadores involucrados deben interactuar por } \\
\text { cierto tiempo, cara a cara, como un equipo auto dirigido } \\
\text { con una meta específica que deben cumplir. } \\
\text { Se da casi de manera imperceptible, pues a partir de }\end{array}$ \\
Transferencia & $\begin{array}{l}\text { la interacción y la interactuación, las personas pueden } \\
\text { generar y transmitir conocimiento tácito. }\end{array}$ \\
Sólo se podrá hacer en aquellos investigadores que se \\
involucraron en el proceso.
\end{tabular}

Fuente: adaptado de Demuner, Nava y Sandoval (2015).

\section{Externalización}

Transita de un conocimiento tácito a un conocimiento explícito. El conocimiento que poseen los investigadores a título personal se traduce en conceptos que integran un conocimiento colectivo. Es la etapa clave de creación de conocimiento; es ella en la que el conocimiento tácito se convierte en conceptos explícitos o comprensibles. Este proceso se realiza en dos fases, en la primera, mediante metáforas se reconocen contradicciones, y en la segunda por medio de analogías, se da solución a las mismas. La externalización se observa típicamente en el proceso de creación de conceptos y es generada por el diálogo o la reflexión colectiva, a través de la propia articulación de éste (Nonaka y Konno, 1998) (Ver tabla 3).

Medios que se pueden emplear para exteriorizar el conocimiento:

a) Utilización de metáfora

b) Uso de analogías

c) Uso de teoremas, hipótesis

d) Modelo 
e) Eslogan

Tabla 3.Proceso de externalización

\begin{tabular}{|c|c|}
\hline Actividad & Descripción \\
\hline Interacción & $\begin{array}{l}\text { Se inicia con un campo de interacción, en el que se ma- } \\
\text { nifiesta un modelo mental (conocimiento tácito) que se } \\
\text { compartirá con otros individuos. }\end{array}$ \\
\hline Reflexión colectiva & $\begin{array}{l}\text { Los involucrados en el proceso mantienen un diálogo } \\
\text { continuo y constante, desarrollando reflexiones colecti- } \\
\text { vas sobre recursos, actividades y mejores prácticas. }\end{array}$ \\
\hline Creación de conceptos & $\begin{array}{l}\text { Se construye paulatinamente y como resultado de cons- } \\
\text { tantes reflexiones colectivas, pues a partir de la interac- } \\
\text { ción y la interactuación el modelo mental tácito que se } \\
\text { ha compartido con otros, se verbaliza en palabras y fra- } \\
\text { ses para aterrizarlo en conceptos explícitos (diagramas, } \\
\text { analogías, esquemas, etc.) }\end{array}$ \\
\hline Validación de conceptos & $\begin{array}{l}\text { Se determina si los conceptos creados son válidos para } \\
\text { todos los casos y productos/servicios solicitados en la } \\
\text { organización. }\end{array}$ \\
\hline Distribución & $\begin{array}{l}\text { Se hace con personas dentro y/o fuera de la organiza- } \\
\text { ción, a partir de una capacitación sobre el lenguaje uti- } \\
\text { lizado. }\end{array}$ \\
\hline
\end{tabular}

Fuente: adaptado de Demuner, Nava y Sandoval (2015).

\section{Combinación}

Es la parte del proceso que sintetiza los conceptos explícitos y los traslada a una base de conocimiento, mediante la captura e integración de nuevo conocimiento explícito esencial, a través de la recopilación, reflexión y síntesis.

Involucra dos actividades esenciales, la primera, es el procesamiento de documentos, estadísticas, informes, minutas, manuales, etc., y la segunda, tiene que ver con la difusión del conocimiento mediante los procesos de transferencia utilizados normalmente por el investigador (Ver tabla 4).

Medios que se pueden emplear para combinar conocimiento:

a) Documentos (Artículos de investigación, libros, resúmenes investigativos, informes técnicos)

b) Congresos, simposios, conferencias 
c) Conversaciones con grupos de investigación, colegas (Vía Internet, teléfono, videoconferencias, etc.)

d) Redes computarizadas de comunicación

Tabla 4. Proceso de combinación

\begin{tabular}{ll} 
Actividad & Descripción \\
\hline Ordenar & $\begin{array}{l}\text { Agrupar por categorías el conocimiento previamente dialogado y refle- } \\
\text { xionado. }\end{array}$ \\
Clasificar & $\begin{array}{l}\text { Contextualizar el conocimiento ya sea por funciones, áreas, disciplinas, } \\
\text { teorías, modelos, etapas del proceso, actividades de la cadena de valor, } \\
\text { de acuerdo al uso que se le valla a dar. } \\
\text { De acuerdo a como fue clasificado, se decidirá cómo documentar el } \\
\text { conocimiento, ya sea con modelos, diagramas, mapas, procedimientos, } \\
\text { manuales, fichas técnicas, folletos, etc. }\end{array}$ \\
Retroalimentar & $\begin{array}{l}\text { Someter a revisión la documentación elaborada para corregir posibles } \\
\text { errores. }\end{array}$ \\
\hline
\end{tabular}

Fuente: adaptado de Demuner, Nava y Sandoval (2015).

\section{Internalización}

De explícito a tácito. Es la etapa del proceso en la que se amplía el conocimiento tácito del investigador a partir del conocimiento explícito de la organización, al depurarse este último y convertirse en conocimiento propio de cada persona.

La internalización requiere por un lado la actualización de los conceptos o métodos explícitos y, por otro, la inclusión de dicho conocimiento explícito en tácito. La condición necesaria es que el conocimiento explícito sea vivido o experimentado, bien, pasando personalmente por la experiencia de realizar una actividad, o bien a través de la participación, de las simulaciones, o de los ejercicios de juego de rol, para que así el individuo lo haga propio según su particular estilo y hábitos. Para que el conocimiento explícito se vuelva tácito, es de gran ayuda que el conocimiento se verbalice o diagrame en documentos, manuales o historias orales (Ver tabla 5).

Medios que se pueden emplear para internalizar el conocimiento:

a) La documentación ayuda a apropiarse lo que han experimentado, enriqueciendo su conocimiento tácito. 
b) Los manuales facilitan la transferencia de conocimiento explícito a otras personas, permitiendo que experimenten indirectamente las vivencias de otros, es decir, que las re experimenten (por ejemplo, documentar posiciones a favor o en contra de la línea o tema de investigación puede ser utilizada por los miembros del mismo grupo de investigación).

c) Se llevan a cabo modelos mentales compartidos.

d) Las experiencias y procedimientos aplicados se conservan en memorias documentales u otros medios.

e) Uso de otros métodos propios.

Tabla 5. Proceso de internalización

\begin{tabular}{ll} 
Etapa & Descripción \\
\hline Introducción & $\begin{array}{l}\text { Se "presenta" el conocimiento proveniente de una investigación a un } \\
\text { nuevo receptor de la información }\end{array}$ \\
Interpretación & $\begin{array}{l}\text { El nuevo receptor del conocimiento lo interpreta adaptándolo a sus } \\
\text { ideas, modelos o esquemas antecedentes y lo re interpreta. }\end{array}$ \\
Comprensión & $\begin{array}{l}\text { Al poner en "práctica" el conocimiento interpretado, amplía su com- } \\
\text { prensión, buscando mayor cantidad de información u otros conocimi- }\end{array}$ \\
& $\begin{array}{l}\text { entos complementarios que permitan extender su idea } \\
\text { Explicación }\end{array}$ \\
& $\begin{array}{l}\text { Al poder "explicar" al otro, a través de metáforas, nuevas ideas, es- } \\
\text { conocimiento, haciéndolo suyo y por tanto lo ha internalizado }\end{array}$ \\
\hline
\end{tabular}

Fuente: adaptado de Demuner, Nava y Sandoval (2015).

\section{Reflexiones Finales}

Difundir el conocimiento es responsabilidad de todo invstigador. Para crear, identificar, coleccionar, adaptar, aplicar, organizar y finalmente compartir una serie de conocimientos tácitos y explícitos que pueden permitir el mejor desarrollo (Del Moral et al., 2007) de un documento de investigación se propone socializar el conocimiento con la creación de un campo de interacción que permite que un equipo de colegas compartan sus experiencias y modelos mentales.

En segundo lugar, la externalización se da a partir de un diálogo o reflexiones colectivas significativas, en los que el uso de una metáfora o una analogía apropiadas ayudan al investigador a enunciar el conocimiento tácito oculto, que de otra manera resulta difícil de comunicar. 
En tercer lugar, la combinación da comienzo con la distribución por redes del conocimiento recién creado y el conocimiento existente en el grupo, cristalizándolos así en un nuevo producto de investigación, en esta etapa se formaliza lo investigado.

$\mathrm{Y}$ en cuarto lugar, la internalización se origina en aprender haciendo, se da a conocer, se difunde el conocimiento, pero éste no termina en la cuarta etapa, en ella surgen nuevas inquietudes, perspectivas, avances, dominios que hacen que el proceso se re inicie con la socialización del conocimiento.

La creciente presión por publicar e incrementar el número de citas de investigación interdisciplinaria, ha hecho que los estudios sean estadísticamente significativos, pero teóricamente fragmentados (Tsang y Kwan, 1999), un problema común en la mayoría de las áreas de investigación de la organización (Lane, Koka y Pathak, 2006).

En este sentido, el uso del método descrito anteriormente debe verse como un aliciente para el investigador, de tal forma que le motive a realizar aportaciones de calidad. Y aunque existe la presión por la publicación que exigen organismos que fomentan y respaldan la investigación, el objetivo no es lograr una cantidad, sino calidad en las aportaciones que tanto hacen falta a la ciencia básica y aplicada, sobre todo a las ciencias sociales.

\section{Referencias}

Bedrow, H.W.L. (2003). International Joint Ventures: Creating Value through Successful Knowledge. Management, Journal of World Business, 38(1): 15-30.

Byosiere, P. (1999). Fusión y difusión de las esferas de conocimiento en el ámbito regional, Las sociedades del conocimiento (pp. 81-86), editado por Cluster del Conocimiento, Zamudio.

Del Moral, A.; Pazos, J.; Rodríguez, E.; Rodríguez-Patón, A. \& Suárez, S. (2007). Gestión del Conocimiento (pp. 499). Madrid, España: Thomson.

Demuner, F.M.R.; Nava, R.R.M. \& Sandoval, A.R. (2015). TI en la dinàmica del conocimiento empresarial. México: Bonobos Editores.

Fisher, R.S. \& Powers, L.E. (2004). Peer-Review. Publication: a view from inside. Epilepsia, 45(8): 889-894.

Grawitz, M. (1974). Méthodes des sciences socials. Paris: Dalbor. 
Lane, P.J.; koka, B.R. \& Pathak, S. (2006). The reification of absorptive capacity: a critical review and rejuvenation of the construct. Academy of Management Review, 31(4): 833-863.

Nonaka, I. \& Takeuchi, H. (1995). The Knowledge-Creating Company. How Japanese companies create the dynamics of innovations. New York, NY: Oxford University Press.

Nonaka, I. \& Konno, N. (1998). The Concept of "Ba, Building a Foundation for Knowledge Creation. California Management Review, 40(3), Spring.

Osterloh, M. \& Frey, B.S. (2000). Motivation, Knowledge Transfer, and Organizational Forms. Organization Science, Sep.-Oct., 11(5): 538-550. Stable URL: http://links.jstor.org

Tsang, E. \& Kwan, K. (1999). Replication and theory develop- ment in organizational science: A critical realist perspective. Academy of Management Review, 24: 759-780.

Xavier, B.F.A. \& Bermejo, J. (2002). ¿Por qué se ha rechazado nuestro artículo?. Revista Española de Cardiología, 55(07), www.revespcardiol.org 